\title{
High-lights of the ATLAS and ALICE experiments
}

\author{
Tancredi Carli* ${ }^{*}$ \\ CERN \\ E-mail: tancredi.carliecern.ch \\ On behalf of the ATLAS and ALICE collaborations
}

The ATLAS experiment has achieved important new physics results in particular in the Higgs area using the large proton-proton collision data-sets at a centre-of-mass energy of $13 \mathrm{TeV}$. The Yukawa coupling of the Higgs boson to fermions was established with the direct observation of the production of the Higgs boson with a top quark pair and the observation of the Higgs boson decay to beauty quarks. The four main production modes of the Higgs boson have now been observed. The couplings to all heavy particles (Z/W-boson, top and bottom quark and tau-lepton) are observed and already measured with good precision. It is experimentally confirmed that the Higgs boson couplings strongly depend on the particle mass.

ATLAS also observed a few important electroweak diboson processes and measured their crosssection and some properties. For some of these processes it is the first observation by the ATLAS experiment. A preliminary measurement of the electroweak mixing angle accomplished a 15\% precision using Drell-Yan cross-section measurements at a centre-of-mass energy of $8 \mathrm{TeV}$. Measurements of spin correlations between top quark pairs show that a discrepancy with NLO QCD. New measurements of top quark pair production in association with beauty quark pairs show a $50 \%$ normalisation difference with respect to most recent QCD calculations.

The ALICE experiments studies particle production in proton-proton and heavy-ion collisions in great detail. New results on strange particle production and baryon-to-meson ratio lead to a consistent picture in proton-proton, xenon-xenon and lead-lead collisions. Models of collective flow measurements tuned in lead-lead collisions can also describe the xenon-xenon data showing that the initial matter distributions in the collisions and the expansion dynamics of the hot and dense nuclear medium are well understood. Moreover, detailed measurements of D-meson demonstrate a quantitative understanding of charm transport.

The 39th International Conference on High Energy Physics (ICHEP2018)

4-11 July, 2018

Seoul, Korea

\footnotetext{
* Speaker.

${ }^{\dagger}$ Many thanks to the ATLAS and ALICE collaborations for preparing excellent results on the latest data-sets for this conference and for the help to prepare this invited plenary talk.
} 


\section{Progress in Higgs physics}

A new particle with a mass of $125 \mathrm{GeV}$ was discovered in July 2012 by the ATLAS and the CMS experiments at LHC using proton-proton collisions at a centre-of-mass energy of $\sqrt{s}=7$ and $8 \mathrm{TeV}$ (Run-1) [1]. The large data-sets collected at $\sqrt{s}=13 \mathrm{TeV}$ (Run-2) enable detailed measurements of the newly discovered particles that can answer the question: Is this particle the Higgs boson as assumed in the Standard Model (SM) ?

To describe the Higgs interactions for the electro-weak breaking mechanism the SM extends the Lagrangian describing the strong and electro-weak interactions. One new term describes the interaction of a scalar $(\Phi)$ with gauge bosons $\left(\left|D_{\mu} \Phi\right|^{2}\right)$. Such an interaction is new for a scalar, but was already known for fermions $(\Psi)$. A completely new interaction type is the Yukawa coupling of the new scalar with fermions $\left(\Psi_{i} y_{i j} \Psi_{j} \Phi\right)$. These two new terms are experimentally tested with the ATLAS data. The Higgs potential $V(\Phi)=\mu^{2} \Phi^{2}+\lambda \Phi^{4}$ can only be explored at the high luminosity LHC.

With the data-set collected in the years 2015 and 2016 corresponding to an integrated luminosity of $L=36 \mathrm{fb}^{-1}$ and in addition with the one including also the 2017 data $\left(L=80 \mathrm{fb}^{-1}\right)$ many new detailed measurements by the ATLAS experiment confirm that the new scalar behaves like the SM Higgs boson.
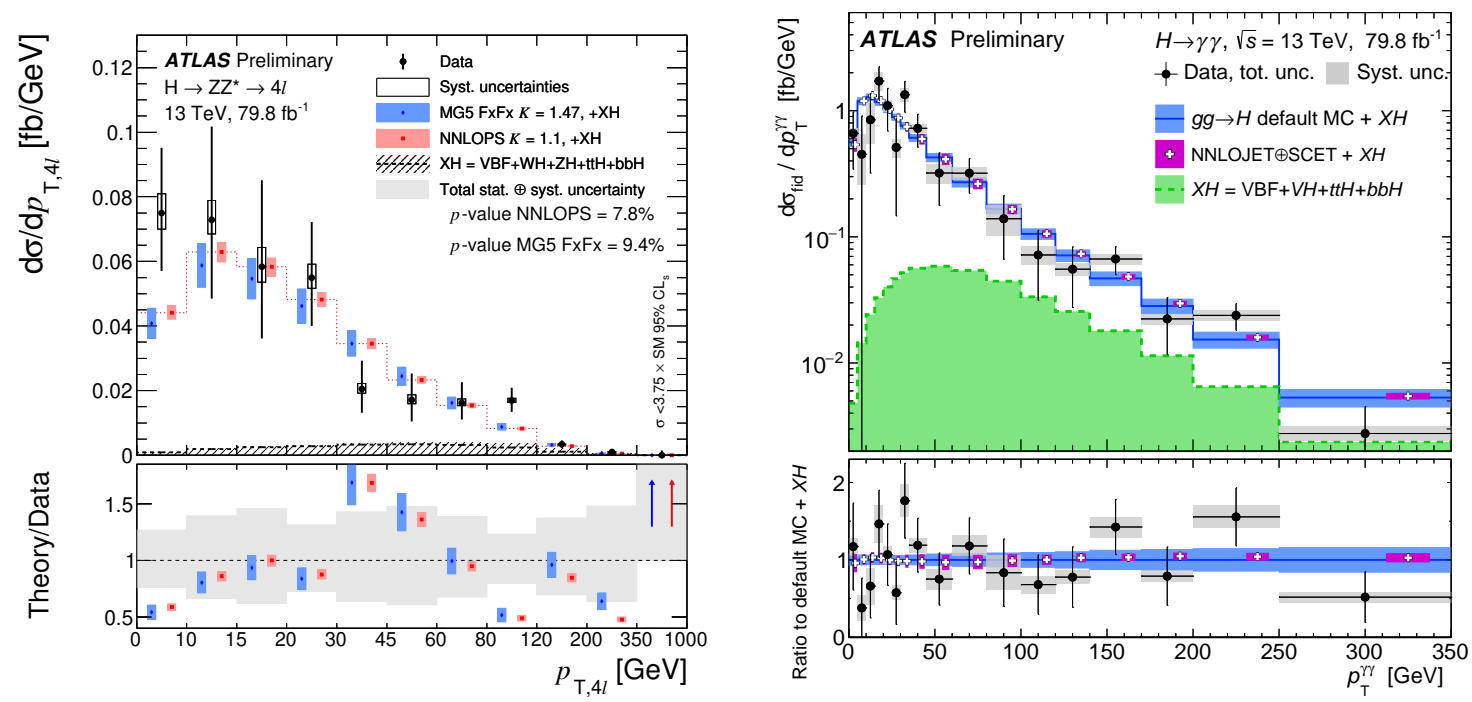

Figure 1: Differential fiducial cross sections for the transverse momentum of the Higgs boson in the four lepton [2] (left) and the two photon [3] (right) channel.

Higgs interactions with gauge bosons These interactions have a high cross-section or clean experimental signatures. The main channels $\left(H \rightarrow \gamma \gamma, H \rightarrow Z Z^{*}\right.$ and $\left.H \rightarrow W W^{*}\right)$ lead to the Higgs discovery in Run-1. A first cross-section measurement was recently made for $H \rightarrow W W^{*}[4,5]$ at $\sqrt{s}=13 \mathrm{TeV}$. The gluon-gluon fusion (ggF) production cross-section $\sigma_{H \rightarrow W W^{*}}^{\mathrm{ggF}}$ is measured with a precision of $17 \%$. Gauge interactions are also used to measure precise fiducial differential crosssections shown for the four lepton channel ( $p p \rightarrow H \rightarrow Z Z \rightarrow l l l l$ [2] and the two photon channel [3] ( $p p \rightarrow H \rightarrow \gamma \gamma)$ in Fig. 1. The data are well described by recent SM predictions based on state-of-the art QCD calculations. 
Yukawa coupling to fermions The analyses of the Run-1 data-set only got a glimpse on the Yukawa couplings to fermions. The combined result of the ATLAS and the CMS experiments gave an observation of the $H \rightarrow \tau \tau$ decay with a significance of 5.5 (5.0) $\sigma$ obs (exp) [6]. With the 36 $\mathrm{fb}^{-1}$ data-set the ATLAS experiment alone has reached 6.4 (5.4) $\sigma$ obs (exp) by combining the 7,8 and $13 \mathrm{TeV}$ data-sets. The precision of the fiducial cross-section is $50 \%$ for the ggF process and $60 \%$ for the Vector-boson fusion process (VBF). The measurements of $H \rightarrow \tau \tau$ and $H \rightarrow W W$ require a detailed understanding of all backgrounds and are experimentally challenging.

A very important milestone in Higgs physics was achieved this year with the direct observation of the associated production of the Higgs boson with a top quark pair $(t \bar{t} H)$. This directly confirms the large Yukawa coupling $\left(y_{t}\right)$ is proportional to $m_{t} \sqrt{2} / v \approx 1$, with the top mass $m_{t}$ and the vacuum expectation value of the Higgs field $v=246 \mathrm{GeV}$.

In December 2017 the ATLAS experiment reported evidence for $t \bar{t} H$ production with a combined significance of 4.2 (3.8) $\sigma$ observed (expected) [7, 8, 9]. Including the 2017 data for the channels $t \bar{t} H(\rightarrow \gamma \gamma)$ and $t \bar{t} H\left(Z Z^{*} \rightarrow 4 l\right) t \bar{t} H$ production was observed with a significance of 5.1 (6.3) $\sigma$ observed (expected) [10]. The signal to background yields $(\log (S / B))$ are displayed in Fig. 2 (left). At high $\log (S / B)$ values a clear increase over the background is observed.
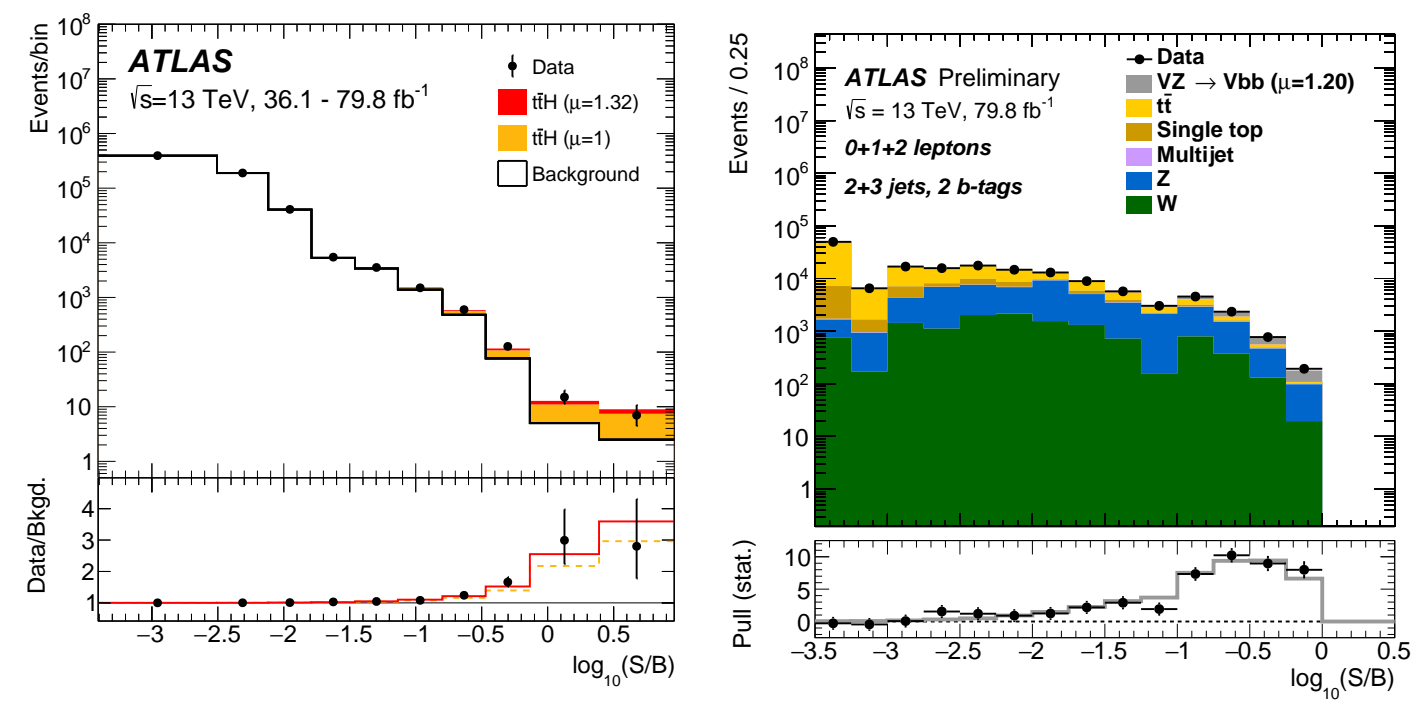

Figure 2: Observed event yields in up to $80 \mathrm{fb}^{-1}$ of $13 \mathrm{TeV}$ data for $t \bar{t} H$ production [10] (left) and $H \rightarrow b \bar{b}[11]$ (right) as a function of the signal to background ratio.

Assuming the SM branching fractions the total $t \bar{t} H$ production cross section at $13 \mathrm{TeV}$ is measured to be $670 \pm 90$ (stat.) ${ }_{-100}^{+110}$ (syst.) fb in agreement with the SM prediction. A 20\% precision is achieved. The observation of the $t \bar{t} H$ process gives direct evidence that the Higgs boson couples directly to the top quark in contrast to the gluon-gluon fusion production mode where the top quarks are in a virtual loop (and therefore might contain new physics contributions). It is therefore interesting to compare the effective coupling of the Higgs boson to gluons via the virtual top loop ( $\left.\kappa_{\text {gluon }}\right)$ and the direct coupling to top quarks $\left(k_{\text {top }}\right)$. The ratio of the effective couplings gives $\kappa_{\mathrm{gluon}} / k_{\text {top }}=1.09 \pm 0.14$ consistent with the SM expectation [12]. Already a remarkable precision of $13 \%$ is achieved. This result limits new physics contributions in the top quark loop. 
The second high-light prepared for this conference is the observation of the associated WH and $\mathrm{ZH}$ production $(\mathrm{VH})$ mode and the Higgs decay in beauty quarks $H \rightarrow b \bar{b}$. This is a key measurement for the Higgs sector since the SM predicts a large branching fraction of 58\%. and also tests the Yukawa coupling to fermions. It also constitutes the first observation of the Higgs boson decaying to quarks. Despite the dominant branching ratio, the observation is very challenging because of the very large backgrounds coming from $\mathrm{W}$ and $\mathrm{Z}$ production in association with bottom and charm quarks. Sophisticated analysis techniques and excellent modelling of the backgrounds are therefore required.

Production mode

Gluon-gluon fusion (ggF)

Ass. WH or $\mathrm{ZH}$ production (VH)

Vector-boson fusion (VBF)

Associated $t \bar{t} H$ production $(t \bar{t} H)$

Table 1: Significance of the main Higgs production modes.

Significance observed in Run-1 $5.3(4.8)$ obs (exp) [11] 6.5 (5.3) obs (exp) [12] $6.3(5.1)$ obs $(\exp )[10]$ $\log (\mathrm{S} / \mathrm{B})$, with $\mathrm{S}$ being the fitted signal and B the fitted background yields. In the lower panel, the pull of the data relative to the background (the statistical significance of the difference between data and fitted background) is shown with statistical uncertainties only. A clear signal is seen at high signal to background ratio.

Using the VH production mode alone a significance of 4.9 (4.3) $\sigma$ obs (exp) is obtained at $\sqrt{s}=13 \mathrm{TeV}$. Combined with all production modes (VBF, $t \bar{t} H, \mathrm{VH})$ and with earlier data at $\sqrt{s}=7$ and $8 \mathrm{TeV}$ a significance of $5.4(5.5) \sigma$ obs (exp) is obtained [11,13]. This constitutes the observation of Higgs decays to beauty quarks! The significance of the production modes are summarised in Tab. 1. All major Higgs production modes are observed.

\begin{tabular}{cc} 
Higgs coupling & Significance/Limit \\
\hline$H \rightarrow Z Z^{*}$ & Run-1 established, Run-2 d $\sigma$ [2] \\
$H \rightarrow W W^{*}$ & 6.3 (5.2) obs (exp) (Run-2 only) [4] \\
Top Quark: $t \bar{t} H$ & $6.3(5.1)$ obs (exp) [10] \\
Beauty-quark: $H \rightarrow b \bar{b}$ & $5.4(5.5)$ obs (exp) [11] \\
Tau-lepton: $H \rightarrow \tau \bar{\tau}$ & $6.4(5.4)$ obs (exp) [14] \\
Muon: $H \rightarrow \mu \bar{\mu}$ & $\sigma_{\text {limit }} / \sigma_{\mathrm{SM}}<2.1$ obs [15] \\
Charm-quark: $H \rightarrow c \bar{c}$ & $\sigma_{\text {limit }} / \sigma_{\mathrm{SM}}<104$ obs [16] \\
\hline
\end{tabular}

With the new results presented at this conference. the couplings to all high mass particles are measured. The next challenge will be to establish the couplings to the lighter particles like muons and charm-quarks. quarks. For those so far only

Table 2: Summary of the Higgs coupling measurements. limits on the production crosssection are available. Increased precision and detailed measurements will be obtained with the large Run-2 data-sets.

One of the next major goals in the LHC physics program is the observation of Di-Higgs production and the extraction of the Higgs selfcoupling. Di-Higgs production directly probes the SM trilinear coupling. Unfortunately in the SM there is a strong destructive interference between the process where each of the two Higgs

\begin{tabular}{ll} 
Channel & $\sigma_{\text {limit }} / \sigma_{p p \rightarrow H H}^{\mathrm{SM}}$ \\
\hline$b \bar{b} \tau^{+} \tau^{-}$ & $12.5(14.8)$ obs (exp) [17] \\
$b \bar{b} b \bar{b}$ & $13.0(20.7)$ obs (exp) [18] \\
$b \bar{b} \gamma \gamma$ & $22.0(28)$ obs (exp) [19] \\
$W^{+} W^{-} \gamma \gamma$ & $230(160)$ obs (exp) [20] \\
\hline
\end{tabular}

Table 3: Di-Higgs cross-sections limits. boson couples to a top quark and the one which involves the Higgs self-coupling (via $H^{*} \rightarrow H H$ ). 
The cross-section is therefore only about $\sigma_{p p \rightarrow H H}^{\mathrm{SM}}=34 \mathrm{fb}$. The present analyses could already be sensitive to new physics contributions and therefore ATLAS carries an active program with many decay modes analysed. Recently a lot of progress was made. In particular, the $H H \rightarrow \bar{b} b \tau^{+} \tau^{-}$ result has been considerably improved [17] with the upper limit on the cross-section approaching $\sigma_{\text {limit }} / \sigma_{p p \rightarrow H H}^{\mathrm{SM}} \approx 10$. All results are summarised in Tab. 3 .

\section{Observation of rare electro-weak Diboson processes}

The electro-weak production of two bosons tests the electro-weak symmetry breaking, since the Higgs boson leads to a strong cross-section suppression via the cancellation of individual electro-weak diagrams. The $2015+2016$ data-set opened up the possibility to measure several previously unobserved rare electro-weak processes.

The electro-weak production mode is enhanced over the strong production mode in vectorboson scattering topologies (VBS) where a W-boson is radiated from an incoming quark in each colliding proton. This topology has two jets with large invariant mass in the forward detector region. The production of $W^{ \pm} W^{ \pm}$jet jet is particularly sensitive, since the requirement of a samesign W-boson pair gives an additional background suppression. ATLAS observed this process with a significance of $6.9 \sigma$ [21].

\begin{tabular}{ccc} 
Channel & Significance & $\Delta \sigma / \sigma_{\text {fid }}$ \\
\hline Long. W polarisation & $4.2(3.8)$ obs $(\exp )$ & $5 \%[21]$ \\
$W Z$ jet jet & $5.6(3.3)$ obs $(\exp )$ & $26 \%[22]$ \\
$W^{ \pm} W^{ \pm}$jet jet & $6.9(4.6)$ obs $(\exp )$ & $20 \%[23]$ \\
\hline
\end{tabular}

Table 4: Rare electro-weak SM processes measurements.

$W Z$ jet jet production was observed with a significance of 5.6 $\sigma$ and a fiducial cross-section was measured [21] with a precision $\Delta \sigma / \sigma_{\text {fid }}=26 \%$. This process is sensitive to triple and quartic electro-weak gauge couplings. The $\mathrm{Z}$ boson polarisation was measured and evidence for the longitudinal $\mathrm{W}$ boson polarisation was obtained $(4.2 \sigma)$.

Inclusive electro-weak WZ production measurements are an important tool to study QCD and electro-weak effects. The fiducial WZ production cross-sections measured differentially as function of several kinematic variables are in good agreement with NNLO QCD [21]. The longitudinal and transverse W- and Z-boson polarisation in WZ production is measured. Evidence for longitudinal polarisation of the W-boson is found. The results are summarised in Tab. 4.

\section{Precision measurement of electro-weak parameters}

In NNLO QCD the triple differential Drell-Yan cross-section $d \sigma_{p p \rightarrow Z} / d y^{l l} d m^{l l} d \cos \theta$ (dilepton mass and rapidity and polar angle in the lepton rest frame) can be expanded as a sum of nine harmonic polynomials. The angular coefficient $A_{4}$ is parity violating and is particularly sensitive to the electro-weak mixing angle. Experimentally it is closely related to the forward-backward asymmetry. The measurement of the angular coefficients using electrons and muons in the central rapidity $|\eta|<2.4$ (CC) and using electrons in the forward rapidity $2.5<|\eta|<4.6$ (CF) detector regions can be used to determine the effective electro-weak mixing angle. The lepton invariant mass is required to be close to the $\mathrm{Z}$ mass pole to enhance electro-weak effects. 


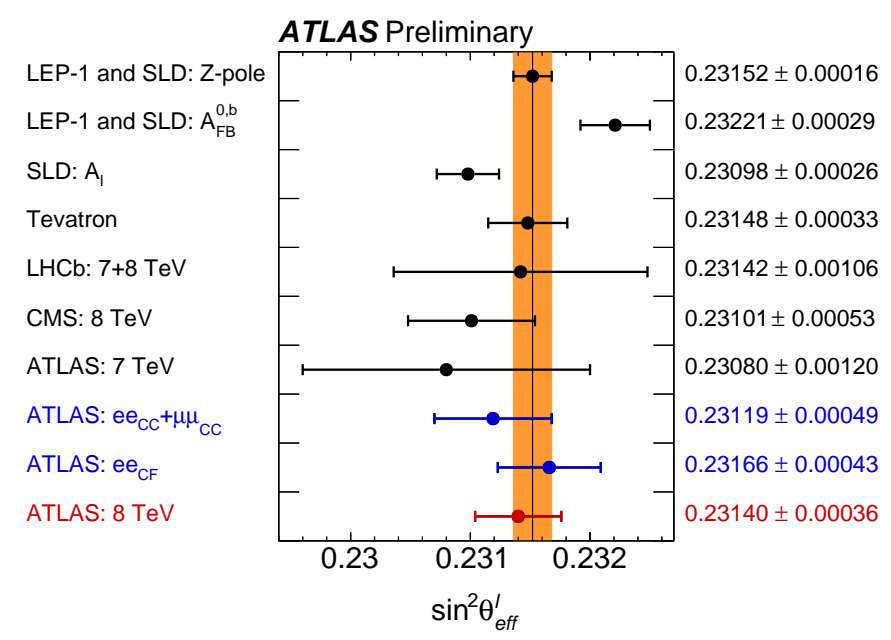

Figure 3: Eff. weak mixing angle measurements [24].
The combined measurement ( $\mathrm{CC}$ and CF) with the $\sqrt{s}=8 \mathrm{TeV}$ data taken in 2012 achieves a $0.15 \%$ precision (see Tab. 5). The largest uncertainty is due to the parton distribution in the proton which reflects the limited knowledge of initial quark direction due to radiation [24]. Fig. 3 summarise the new ATLAS measurements together with earlier measurements from CMS, the Tevatron, LEP and SLD experiments. ATLAS reaches similar precision as previous experiments.

Tab. 5 summarises recent precision measurements of electro-weak parameters by ATLAS.

\section{Top physics}

Since several years ATLAS has measured fiducial top quark production cross-sections defined using the particles entering the detector. Together with the recent improvements of the Monte Carlo event generators giving NLO precision for $2 \rightarrow 2$ with $2 \rightarrow 3$ partons scatterings these measurements have significantly improved the modelling of the final state with top quarks [29]. A fiducial cross-section measurement of $t \bar{t} b \bar{b}$ production shows that the shapes of the differential cross-sections (e.g. as a function of the number of b-jets) are well described, but the absolute cross-section are 50\% higher in the data than in the theory predictions [30].

A measurement of the azimuthal angle between the two leptons in the process $p p \rightarrow t \bar{t} \rightarrow e \mu b \bar{b}$ $(\Delta \Phi(e \mu))$ shows a steeper dependence in data compared to recent Monte Carlo generators [31]. This variable is particularly sensitive to spin correlations between the top decay products. If the discrepancy is interpreted as due to spin correlations, stronger spin correlations than expected by NLO QCD and Monte Carlo generators are observed in data. The observed discrepancy is on the level of $3.2 \sigma$.

\section{Searches for Physics Beyond the Standard Model}

ATLAS has a very active physics program on searches for new particles and interactions beyond the SM. Examples are searches for super-symmetric particles, new additional Higgs bosons, Dark Matter candidates or new weak interactions. ATLAS published 62 articles on searches in the $13 \mathrm{TeV}$ data-set with $\mathrm{L}=36 \mathrm{fb}^{-1}$ and there are already 13 preliminary results based on the 2017 with $\mathrm{L}=80 \mathrm{fb}^{-1}$.

An example is a search for a new electro-weak gauge boson $\left(W^{\prime}\right)$ in context of sequential SM benchmark model [32]. The $W^{\prime}$ mass is reconstructed from a lepton at high transverse momentum 


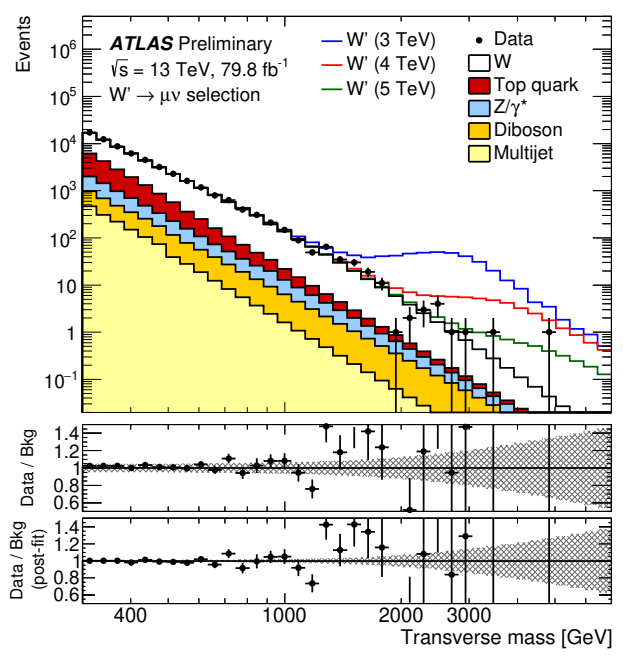

(a)

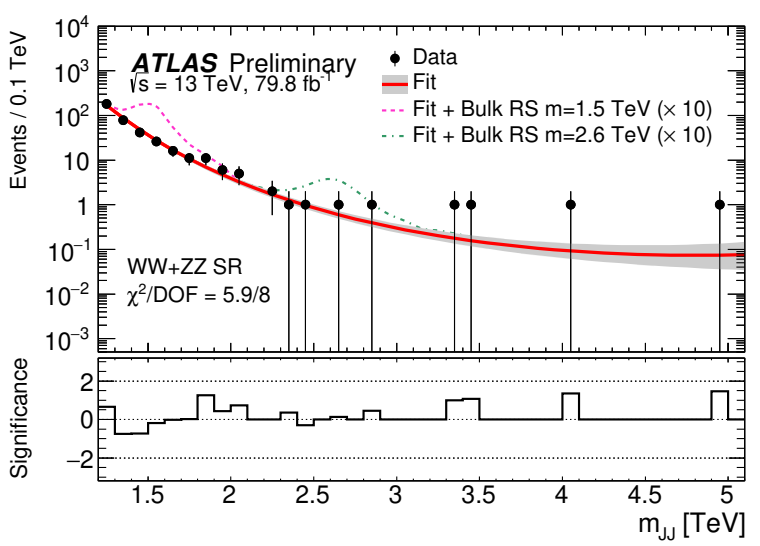

(b)

Figure 4: Mass spectra for the $W^{\prime}$-boson [32] (a) and the Diboson resonance search [33] (b).

and the missing transverse momentum (see Fig. 4a). Masses up to about $3 \mathrm{TeV}$ are observed, but no deviation from the background is found. Assuming SM couplings $W^{\prime}$ masses below $5.6 \mathrm{TeV}$ can be excluded at $95 \% \mathrm{CL}$ with $\mathrm{L}=80 \mathrm{fb}^{-1}$ [32], while masses below $5.2 \mathrm{TeV}$ where already excluded with the $36 \mathrm{fb}^{-1}$ data-set [34]. This demonstrates that the pure gain due to a luminosity increase is becoming less important in the future. However, new signatures and new experimental techniques can further increase the sensitivity to find new physics.

A nice example is the search for resonances decaying in two $\mathrm{W}$ bosons at high transverse momentum. The invariant mass of two boosted $\mathrm{W}$ bosons reconstructed by two large radius jets is shown in Fig. 4. No new resonance is observed. A new experimental technique considerably improved the W-boson tagging performance by using tracks to define the direction of the jet constituents instead of calorimeter clusters. Together with a working point optimisation an improvement by a factor of 2 beyond the gain by the increased luminosity has been achieved with respect to earlier results based on the $36 \mathrm{fb}^{-1}$ data-set [33].

\section{Heavy Ion physics with the ALICE experiment}

The main ALICE results are based on lead-lead (Pb-Pb) and p-Pb collisions. In 2017 six LHC hours were used for xenon-ion (Xe) collisions. This year a significant increase in $\mathrm{Pb}-\mathrm{Pb}$ luminosity is expected to study rare processes.

The ALICE detector with its impressive particle-identification capabilities can measure the production yields of $K_{S}, \Lambda, \Phi, \Theta$ and $\Omega$ particles with respect to pion yields as a function of the mean charged particle multiplicity in various collision set-ups (see Fig. 5). A smooth evolution of the strange baryon-to-pion ratios as a function of charged particle multiplicity is observed. They increase smoothly until a plateau is reached. This trend is confirmed by the recent Xe-Xe data.

The right panel of Fig. 5 shows the baryon-to-meson ratios for non-strange, strange and charmed baryons and mesons as a function of transverse momentum. A well pronounced bump is observed around $p_{t} \approx 3 \mathrm{GeV}$ which is difficult to explain with QCD models. The bump was recently confirmed for the $\Lambda_{c}$ to $D^{0}$-meson ratio and the same effect is seen in $\mathrm{p}$ - $\mathrm{p}$ and $\mathrm{p}$ - $\mathrm{Pb}$ collisions. 

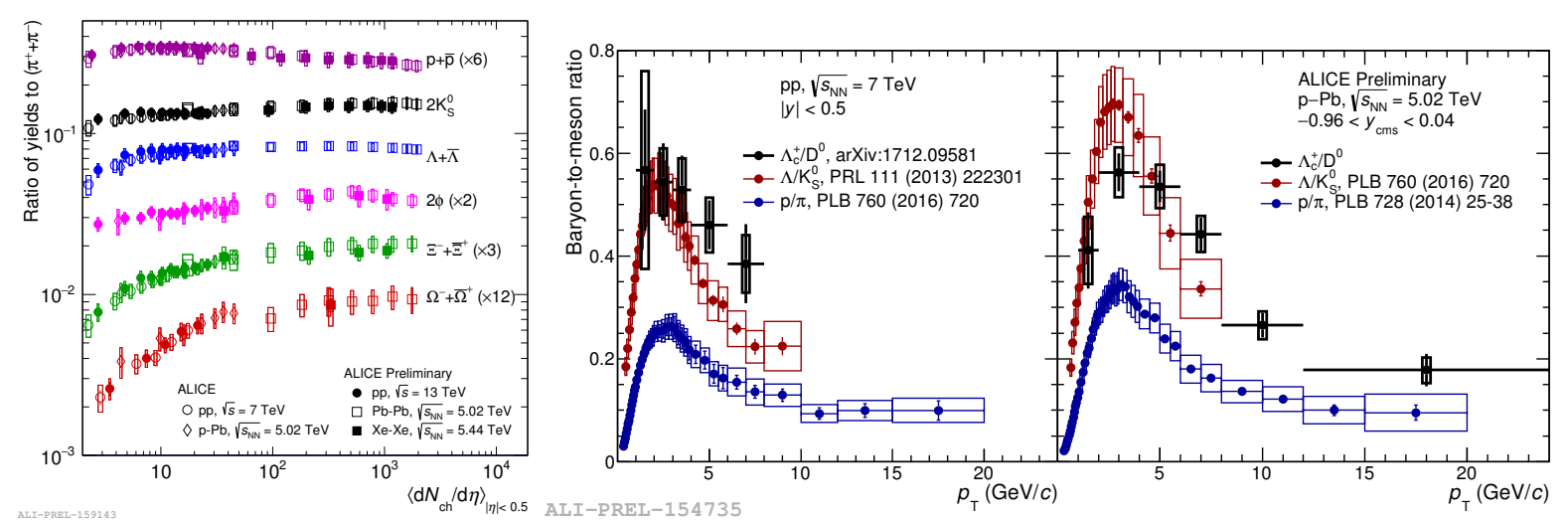

Figure 5: Particle yields for protons, $K_{S}^{0}, \Lambda, \Xi, \Omega$ and $\Phi$ normalised to pion yields versus the average multiplicity in p-p $7 \mathrm{TeV}$, p-p $13 \mathrm{TeV}$, p-Pb $5 \mathrm{TeV}, \mathrm{Pb}-\mathrm{Pb} 5 \mathrm{TeV}$ and $\mathrm{Xe}-\mathrm{Xe} 5.44 \mathrm{TeV}$ collisions [35] (left). Baryon-to-meson ratio $\left(\Lambda_{c}^{+} / D^{0}, \Lambda^{0} / K_{s}, p / \pi\right)$ vs $p_{t}$ in $\mathrm{p}-\mathrm{p}$ and $\mathrm{p}$-Pb collisions

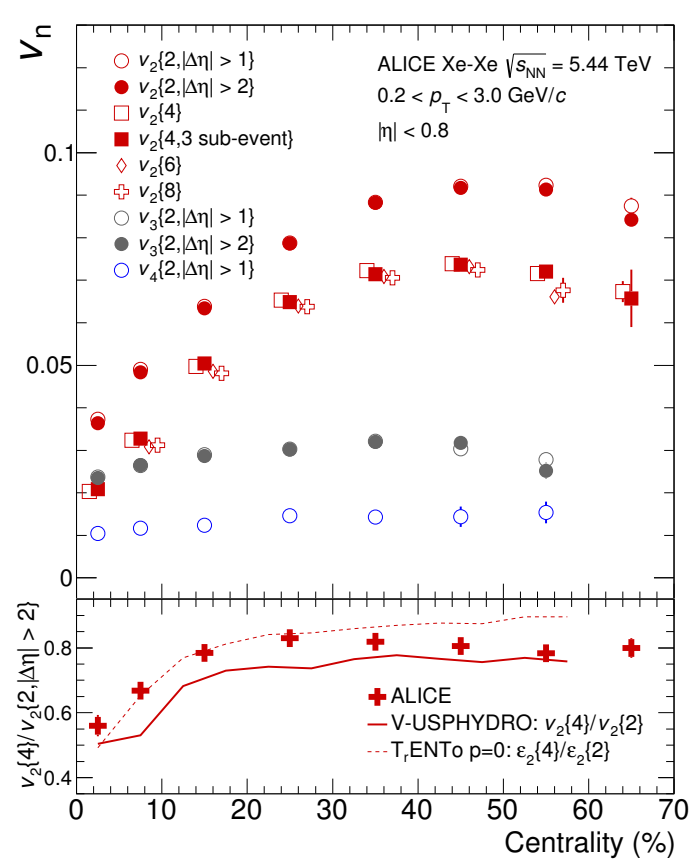

Figure 6: Charged particle elliptic and triangular flow integrated over the range $0.2<p_{T}<3.0 \mathrm{GeV}$ as a function of centrality in Xe-Xe collisions [37].
The collective movement of particles produced in heavy ions collisions gives important insights into the particle formation mechanism in early stages of the nucleon interactions. The production of particles shows an azimuthal anisotropy originating from the non-isotropic density distribution in the initial state which leads to anisotropic expansion of the hot and dense matter. The final state azimuthal anisotropy is characterised by the harmonic coefficients $v_{n}$ of a Fourier expansion of the azimuthal distributions. The $v_{2}$ coefficient is called elliptic flow. It is dominant for non-central collisions where the initial collision area has an elliptic shape; $v_{3}$, or triangular flow is the result of fluctuations in the initial state causing triangular components.

Fig. 6 shows $v_{2}$ and $v_{3}$ as function of the centrality in Xe-Xe collisions at $\sqrt{s_{N N}}=5.44 \mathrm{TeV}$ [37]. The elliptic flow increases from low centrality where the collisions are head-on to large values where the nuclei only collide at the periphery. The model calculations tuned on $\mathrm{Pb}-\mathrm{Pb}$ results are in good agreement with the Xe-Xe measurements. This demonstrates that the effect of the initial density distributions and the viscosity on elliptic and triangular flow are well understood. The elliptic flow is studied in more detail in $\mathrm{Pb}-\mathrm{Pb}$ collisions using $D$-mesons as function of the $p_{t}$ in Fig. 7 [38]. The elliptic flow is maximal at $p_{t} \approx 3 \mathrm{GeV}$ and then decreases indicating a strong particle suppression at high $p_{t}$. The azimuthal distribution of initial charm quark production is uniform and the observed anisotropy is due to scattering in the medium. This process can be characterised by the charm quark diffusion coefficient, which these measurements will help to determine. 


\section{Conclusions}

The ATLAS analysis of the Run-2 data is in full swing. Important key Higgs physics results have been obtained this year. The Higgs decay to beauty quarks and the main Higgs production modes are observed. The Higgs coupling to the top quark was directly observed. As a results the Yukawa coupling of the Higgs boson to fermions is experimentally confirmed.

Rare electro-weak process like the same-sign

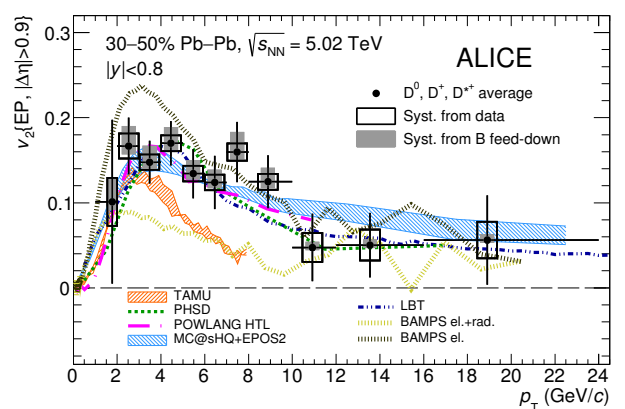

Figure 7: Elliptic flow of $D$-mesons in $\sqrt{s_{\mathrm{NN}}}=5.02 \mathrm{TeV} \mathrm{Pb}-\mathrm{Pb}$ collisions [38]. $W^{ \pm} W^{ \pm}$jet jet and and the $W Z$ jet jet processes were observed. They constitute an important test of the SM electro-weak sector. A new measurement of the electro-weak mixing angle with a precision of $0.15 \%$ completes the recent precise electro-weak parameter measurement of the Higgs, the $W$ and the top mass.

The large data-set also allows for precise measurements of top quark pair final states. Examples are a measurement of associated production with beauty quark pairs and an indication for stronger top spin correlations than expected by NLO QCD.

The extensive and active program for new physics with the Run-2 data-set is under way. The searches on the intermediate data-set do not show any sign for new physics. However, many new directions are being worked out and the detectors are pushed at the limit of their performance to cover all new physics possibilities.

In heavy ion collisions good progress has been made to understand the properties of strongly interacting nuclear matter. Azimuthal anisotropies in Xe-Xe data confirm the understanding of the expanding of the dense and hot nuclear medium. The production of charm-mesons probes partonic interactions and the quantitative understanding of charm transport is progressing.

\section{References}

[1] ATLAS collaboration, Phys. Lett. B716 (2012) 1 [1207. 7214].

[2] ATLAS collaboration, ATLAS-CONF-2018-018, June, 2018.

[3] ATLAS collaboration, ATLAS-CONF-2018-028, June, 2018.

[4] ATLAS collaboration, ATLAS-CONF-2018-004, May, 2018.

[5] ATLAS collaboration, [1808.09054].

[6] ATLAS, CMS collaboration, JHEP 08 (2016) 045 [1606.02266].

[7] ATLAS collaboration, Phys. Rev. D98 (2018) 052005 [1802.04146].

[8] ATLAS collaboration, Phys. Rev. D97 (2018) 072003 [1712. 08891 ].

[9] ATLAS collaboration, Phys. Rev. D97 (2018) 072016 [1712. 08895 ].

[10] ATLAS collaboration, Phys. Lett. B784 (2018) 173 [1806. 00425 ].

[11] ATLAS collaboration, ATLAS-CONF-2018-036, July, 2018.

[12] ATLAS collaboration, ATLAS-CONF-2018-031, July, 2018. 
[13] ATLAS collaboration, Phys. Lett. B786 (2018) 59 [1808 . 08238].

[14] ATLAS collaboration, ATLAS-CONF-2018-021, June, 2018.

[15] ATLAS collaboration, ATLAS-CONF-2018-026, July, 2018.

[16] ATLAS collaboration, Phys. Rev. Lett. 120 (2018) 211802 [1802 . 04329 ].

[17] ATLAS collaboration, Phys. Rev. Lett. 121 (2018) 191801 [1808.00336].

[18] ATLAS collaboration, [1804.06174].

[19] ATLAS collaboration, JHEP 11 (2018) 040 [1807. 04873$].$

[20] ATLAS collaboration, [1807.08567].

[21] ATLAS collaboration, ATLAS-CONF-2018-034, July, 2018.

[22] ATLAS collaboration, ATLAS-CONF-2018-033, July, 2018.

[23] ATLAS collaboration, ATLAS-CONF-2018-030, July, 2018.

[24] ATLAS collaboration, ATLAS-CONF-2018-037, July, 2018.

[25] ATLAS collaboration, Eur. Phys. J. C78 (2018) 110 [1701 . 07240].

[26] ATLAS collaboration, Phys. Lett. B784 (2018) 345 [1806 . 0 0242].

[27] ATLAS collaboration, ATLAS-CONF-2017-071, September, 2017.

[28] ATLAS collaboration, [1810.01772].

[29] ATLAS collaboration, ATLAS-PUB-2018-009, July, 2018.

[30] ATLAS collaboration, ATLAS-CONF-2018-029, July, 2018.

[31] ATLAS collaboration, ATLAS-CONF-2018-027, July, 2018.

[32] ATLAS collaboration, ATLAS-CONF-2018-017, June, 2018.

[33] ATLAS collaboration, ATLAS-CONF-2018-016, May, 2018.

[34] ATLAS collaboration, Eur. Phys. J. C78 (2018) 401 [1706. 04786].

[35] ALICE collaboration. ALI-PREL-159143, July, 2018.

[36] ALICE collaboration. ALI-PREL-154735, May, 2018.

[37] ALICE collaboration, Phys. Lett. B784 (2018) 82 [180 0 . 01832].

[38] ALICE collaboration, JHEP 10 (2018) 174 [1804. 09083 ]. 